\title{
Efectos de un entrenamiento neuromuscular sobre el equilibrio postural dinámico y propiocepción en basquetbolistas juveniles con inestabilidad funcional de tobillo Effects of neuromuscular training on dynamic postural balance and proprioception in youth basketball players with functional ankle instability
}

Karina Vásquez-Orellana; Matías López-Vásquez; Guillermo Méndez-Rebolledo; Eduardo Guzmán-Muñoz Universidad Santo Tomás (Chile)

Resumen. Introducción: La prevención de lesiones depende de varios factores en el deportista, en donde un buen equilibrio postural y propiocepción son claves para lograrlo. Se plantea que el entrenamiento neuromuscular podría mejorar el equilibrio postural dinámico y propiocepción en basquetbolistas con inestabilidad funcional de tobillo (IFT). Método: Ensayo clínico controlado y aleatorizado. La muestra fue compuesta por 27 jugadoras de basquetbol entre 15 a 17 anos, pertenecientes a un Club Deportivo de la comuna de Maipu (Santiago de Chile). 13 de ellas asignadas al grupo control y 14 a un grupo experimental. Este ultimo grupo recibió una intervención neuromuscular de 6 semanas, de 2 días no consecutivos. Se trabajaron ambas extremidades en superficies estables, inestables, con y sin inhibicion visual. Se midió el equilibrio postural con la prueba SEBTm y la propiocepción a través de una prueba de reposicionamiento articular activo. Resultados: tanto el grupo control como experimental mejoraron significativamente el equilibrio postural, no obstante, el grupo experimental evidenció mayores cambios porcentuales y tamaños del efecto (TE) más grandes. El mayor cambio en el grupo control fue de 7,8\% en la dirección posterolateral del SEBTm $(p=0,046 ; \mathrm{TE}=0,74)$. En el grupo experimental el mayor cambio fue de $21 \%$ en la dirección anterior del SEBTm $(p=0,001 ; \mathrm{TE}=2,2)$. En la prueba de reposicionamiento activo no se observaron cambios significativos en el grupo control, mientras que el grupo experimental mejoró hasta un $82,8 \%$ en la articulación de cadera $(p=0,001 ; \mathrm{TE}=2,72)$. Conclusión: Un entrenaiento neuromuscular de seis semanas mejoro el equilibrio postural dinámico y la propiocepcion en basquetbolistas mujeres con IFT.

Palabras clave: balance postural; propiocepcion; entrenamiento neuromuscular, inestabilidad funcional de tobillo; control postural.

Abstract. Introduction: Injury prevention depends on several factors in the athlete, where an optimal postural balance and proprioception are key to achieving it. It is suggested that neuromuscular training could improve dynamic postural balance and proprioception in basketball players with functional ankle instability (FAI). Method: Randomized controlled clinical trial. The sample consisted of 27 female basketball players between the ages of 15 and 17, belonging to a Sports Club in the Maipú city (Santiago, Chile). 13 of them assigned to the control group and 14 to an experimental group. This last group received a neuromuscular intervention of 6 weeks, of 2 non-consecutive days. Both extremities were worked on stable, unstable surfaces, with and without visual inhibition. Postural balance was measured with the SEBTm test and proprioception through an active joint repositioning test. Results: both the control and experimental groups significantly improved postural balance, however, the experimental group showed greater percentage changes and larger effect sizes (ES). The greatest change in the control group was $7.8 \%$ in the posterolateral direction of the SEBTm $(p=0.046 ; \mathrm{ES}=0.74)$. In the experimental group, the greatest change was $21 \%$ in the anterior direction of the SEBTm $(p=0.001$; ES=2.2). In the active repositioning test, no significant changes were observed in the control group, while the experimental group improved up to $82.8 \%$ in the hip joint $(p=0.001 ; \mathrm{ES}=2.72)$. Conclusion: A six-week neuromuscular training improved dynamic postural balance and proprioception in female basketball players with IFT.

Keywords: postural balance; proprioception; neuromuscular training, functional ankle instability; Postural control.

\section{Introducción}

Un esguince lateral de tobillo se produce por una hipersupinación del retropié, típicamente, asociado con una flexión plantar que conduce al estiramiento o desgarro de los ligamentos laterales del tobillo (Fong, Hong,

Fecha recepción: 21-09-21. Fecha de aceptación: 08-02-22

Eduardo Guzman Muñoz

eguzmanm@santotomas.cl
Chan, Yung, \& Chan, 2007). En deportistas, la prevalencia es del 33\% al 80\% (McGuine, Greene, Best, \& Leverson, 2000), siendo más frecuente en basquetbolistas que en otros atletas como futbolistas y voleibolistas (Danes-Daetz, Rojas-Toro, \& TapiaMendoza, 2020). Además, dentro del basquetbol, la lesión a nivel de tobillo corresponde a la más frecuente (Danes-Daetz, Rojas-Toro, \& Tapia-Mendoza, 2020; Toro Román et al., 2020). Se ha reportado que el $40 \%$ de los esguinces progresan a inestabilidad funcional de tobillo 
(IFT) (Yeung, Chan, So, \& Yuan, 1994), concepto utilizado para describir una condición recurrente de esguinces y/o sensación de inestabilidad (Freeman, Dean, \& Hanham, 1965).

La IFT generalmente se ha asociado a dos razones: (a) inestabilidad mecánica debido disfunciones de la articulación y estructuras anatómicas de tobillo, y (b) por inestabilidad funcional que se refiere al déficit de integración sensoriomotora entre la articulación y el sistema nervioso central (Jay Hertel, 2008). La lesión causada por un esguince provocaría una pérdida de la información sensorial de la articulación de tobillo, principalmente, por un déficit propioceptivo (Freeman et al., 1965). Esto afectaría la integración sensoriomotora a nivel cerebral y generaría patrones de activación muscular anormales en las personas con IFT (Jay Hertel, 2008). Estudios han demostrado que las personas IFT han presentado alteración de la propiocepción (Rein, Hagert, \& Sterling-Hauf, 2021), perdida del equilibrio postural (Wenning et al., 2020), disminución en la activación muscular (Fatima, Bhati, Singla, Choudhary, \& Hussain, 2020) y retraso en la respuesta muscular (de Oliveira Menacho et al., 2010; Mendez-Rebolledo, Guzmán-Muñoz, Gatica-Rojas, \& Zbinden-Foncea, 2015), corroborando la teoría sensoriomotora como causa de la inestabilidad.

Se ha propuesto que un déficit propioceptivo puede afectar directamente el reconocimiento de la posición de una articulación (Bruyneel, 2016) y alterar el rendimiento de habilidades motoras como el equilibrio (Peterka \& Loughlin, 2004). En los músculos, tendones y articulaciones se encuentran un tipo de receptores sensoriales denominado propioceptores, los cuales retroalimentan al sistema nervioso central respecto a la tensión muscular, sensación del movimiento y sensación de la posición articular (Bruyneel, 2016). Con respecto a la sensación de la posición articular, se han propuesto diversos métodos de evaluación para esta función de la propiocepción, donde la prueba de reposicionamiento articular activo ha sido utilizada en diversas investigaciones (Karagiannopoulos, Sitler, Michlovitz, Tucker, \& Tierney, 2016; Matsouka et al., 2020; Mendez-Rebolledo et al., 2021; Silva-Moya, Mendez-Rebolledo, Valdes-Badilla, Gomez-alvarez, \& Guzman-Munoz, 2021). Esta prueba mide el error entre una posición diana indicada por el evaluador y la capacidad de reproducir esta posición por la persona evaluada (Iida, Kaneko, Aoki, \& Shibata, 2014; Lubiatowski, Ogrodowicz, Wojtaszek, \& Romanowski, 2019).
Por su parte, el equilibrio postural ha sido definido como una habilidad motora compleja derivada de la interacción de diversos procesos sensoriomotores con la finalidad de controlar el cuerpo en el espacio (Horak, 2006; Peterka \& Loughlin, 2004). Dentro de los sistemas sensoriales que contribuyen a mantener un adecuado equilibrio postural se encuentra la información propioceptiva que, en conjunto a la información proveniente de los sistemas visual y vestibular, es integrada en el sistema nervioso central para asegurar un optimo rendimiento de esta habilidad (Maurer, Mergner, \& Peterka, 2006; Peterka \& Loughlin, 2004). Uno de los métodos más reconocidos para evaluar el equilibrio postural dinámico corresponde al Star Excursion Balance Test (SEBT) o su versión modificada denominado SEBTm (Gribble, Hertel, \& Plisky, 2012). El SEBT dentro de su protocolo evalúa ocho direcciones, mientras que el SEBTm evalúa solo tres direcciones: anterior, posteromedial y posterolateral (Gribble et al., 2012). Estas direcciones han demostrado ser las más sensibles para detectar alteraciones del equilibrio postural y riesgo de lesiones (Hertel, Braham, Hale, \& Olmsted-Kramer, 2006).

Los programas de rehabilitación donde se combinan ejercicios específicos de reeducación propioceptiva y de desarrollo del equilibrio postural desempeñan un papel primordial en el manejo de la IFT (Sánchez Monzó, Fuertes Lanzuela, \& Ballester Alfaro, 2015). En los últimos años, uno de los abordajes que ha presentado resultados más alentadores en el tratamiento de trastornos musculoesqueléticos es el entrenamiento neuromuscular. Un entrenamiento neuromuscular es una combinación de ejercicios propioceptivos, activación muscular y equilibrio postural como parte de un programa integral de rehabilitación y prevención de lesiones (Lin, Delahunt, \& King, 2012). Se ha propuesto que un entrenamiento neuromuscular mejora las respuestas motoras inconscientes al estimular las señales aferentes y los mecanismos centrales responsables del control dinámico de las articulaciones (Lin et al., 2012). Se ha demostrado que el entrenamiento neuromuscular mejora la funcionalidad y disminuye el riesgo de lesión (Steib, Rahlf, Pfeifer, \& Zech, 2017), sin embargo los efectos sobre variables sensoriomotoras han sido escasamente reportadas en deportistas. En voleibolistas con IFT se ha observado que el entrenamiento neuromuscular mejora el equilibrio postural estático después de cuatro semanas de intervención (Guzmán-Muñoz et al., 2019), mientras que en futbolistas un entrenamiento neuromuscular de ocho semanas de duración mejoró el 
equilibrio postural estático y el rendimiento en el salto vertical (Jung, Kang, Lee, Park \& Jeong, 2014).

Por lo tanto, el propósito de este estudio fue establecer los efectos de un entrenamiento neuromuscular sobre el equilibrio postural y propiocepción de miembro inferior en basquetbolistas juveniles con IFT.

\section{Material y metodo}

Este estudio fue un ensayo clínico controlado y aleatorizado. Todas las participantes leyeron y firmaron voluntariamente asentimiento informado, mientras que sus tutores legales autorizaron la participación mediante la firma de un consentimiento informado. La investigación fue aprobada por el comite de etica de la universidad Santo Tomas, Chile ( ${ }^{\circ}$ 64.19).

\section{Participantes}

La muestra fue compuesta por 27 jugadoras de basquetbol entre 15 a 17 anos pertenecientes a un club deportivo de la comuna de Maipu, Chile. Trece de ellas fueron asignadas a un grupo control mientras que catorce fueron sometidas a un entrenamiento de tipo neuromuscular (grupo experimental). Se consideraron los siguientes criterios de inclusion: 1) basquetbolistas con IFT segun el cuestionario Cumberland Ankle Instability Tool (CAIT) (Rodriguez-Fernandez, Rebollo-Roldan, Jimenez-Rejano, \& Gueita-Rodriguez, 2015); 2) ultimo episodio de esguince lateral de tobillo entre tres y 12 meses previos a la investigacion (Guzmán-Muñoz et al., 2019; Mendez-Rebolledo et al., 2015); 3) percibir dolor, inestabilidad y / o debilidad en el tobillo (GuzmánMuñoz et al., 2019; Mendez-Rebolledo et al., 2015). Se excluyeron a las deportistas que presentaron las siguientes caracteristicas durante los ultimos 24 meses: 1) desordenes vestibulares; 2) historia de fractura de tobillo; 3) lesion aguda de miembro inferior; 4) historia de cirugia de miembros inferiores; 5) dolor en cualquier articulación al momento de la evaluacion; 6) aquellos que no cumplieron con al menos el 85\% de las sesiones de intervencion consideradas en el estudio.

\section{Equilibrio postural dinámico}

El equilibrio postural dinámico se evaluó mediante la prueba dinámica SEBTm considerando tres direcciones: anterior, posteromedial (PM) y posterolateral (PL). Cada basquetbolista inició la evaluación en posición bípeda con manos en la cintura. Se instruyó a la participante a alcanzar la mayor distancia posible con miembro inferior. Se evaluó miembro inferior dominante y no dominante, donde para efectos de esta prueba la extremidad evaluada es la que soporta el peso corporal. En cada una de las direcciones las participantes tenían tres intentos, seleccionando para el registro el mejor de ellos (Gribble et al., 2012; Gribble, Kelly, Refshauge, \& Hiller, 2013). Se consideró valido el intento cuando el pie de apoyo no se despegaba del suelo y la participante podía recobrar la posición inicial sin perder el equilibrio luego de realizar el alcance. Para medir las distancias alcanzadas en las 3 direcciones se utilizó una cinta métrica en centímetros (Sanny $\mathbb{R}$, Brasil; precisión de $0,1 \mathrm{~mm})$. El valor final del alcance de la extremidad fue expresado en porcentaje, siendo normalizado a partir de la longitud del segmento con el siguiente cálculo:

$$
\% \text { SEBTm }=\frac{\text { distancia alcanzada }(\mathrm{cm})}{\text { longitud segmento }(\mathrm{cm})} \times 100
$$

La longitud del segmento fue medida considerando la distancia existente entre la espina iliaca anterosuperior hasta el maléolo medial del tobillo (Gribble et al., 2013).

\section{Reposicionamiento articular activo}

Se utilizó la prueba de reposicionamiento activo como herramienta de evaluación para medir la sensación de posición articular, una de las funciones de la propiocepción. Se realizó la medición del error posicional en las articulaciones de cadera, rodilla y tobillo a través de un dispositivoWIMU PROTM (RealTrack Systems, Almería, España) que está integrado por sensores inerciales (cuatro acelerómetros, dos giroscopios y un magnetómetro). Se ubicó al sujeto en una posición cómoda para limitar las compensaciones y con los ojos cerrados para evitar la retroalimentación visual. El evaluador a cargo ubico la articulación en una posición objetivo durante tres segundos y se le solicitó a la participante recordar dicha posición. La posición objetivo para las articulaciones evaluadas fue de $70^{\circ}$ de flexión de cadera, $50^{\circ}$ de flexión de rodilla y $20^{\circ}$ de flexión plantar (Silva-Moya et al., 2021). La literatura sugiere el uso de rangos articulares medios como posición objetivo porque se ha visto que es una posición más desafiante de reproducir que los rangos de movimiento extremos (Bruyneel, 2016; Silva-Moya et al., 2021). A continuación, el evaluador llevó la articulación pasivamente a la posición neutra y se le pidió la participante que reposicionara activamente la articulación en la posición objetivo. Cuando la participante indicó que había alcanzado la posición de referencia, se midió en grados el rango articular alcanzado. Se indicó a las participan- 
tes que realizaran la prueba con la mayor precisión posible y que indicaran verbalmente cuándo se sentían seguros de haber alcanzado la posición objetivo. Para determinar el rendimiento de la sensación activa de la posición articular, se utilizó el error absoluto, que fue la diferencia absoluta entre el ángulo objetivo y el ángulo reproducido del participante. La evaluación se realizó 3 veces y se obtuvo la media del error absoluto en grados.

\section{Entrenamiento neuromuscular}

El grupo experimental fue sometido a un entrenamiento neuromuscular, siendo realizado 15 minutos antes del entrenamiento regular de basquetbol en el gimnasio del club deportivo. La intervención neuromuscular tuvo una duración de 6 semanas y se basó en principios utilizados en investigaciones previas (Guzmán-Muñoz et al. 2020; Guzmán-Muñoz et al., 2019; Silva-Moya et al. 2021). Se distribuyó en 2 sesiones semanales de 10 a 15 minutos, en días no consecutivos, totalizando 12 sesiones. La intensidad de entrenamiento fue de carácter progresivo y se centró, principalmente, en la extremidad inferior. Este entrenamiento fue realizado previo el entrenamiento regular de las basquetbolistas. Todas las sesiones contemplaron circuitos con estaciones de trabajo de 30 segundos que consistían en ejercicios de coordinación en escaleras de suelo, saltos en vallas, carrera con cambios de dirección y ejercicios de equilibrio unipodal sobre discos de freeman y mini bosus. Estos ejercicios fueron combinados con actividades de precisión con balones de basquetbol. Cada deportista debía efectuar tres series del circuito completo por sesión, considerando ambas extremidades en los casos de ejercicios unipodales. La primera semana de realizo un entrenamiento con trabajos bipodales en superficies estables. La segunda semana los ejercicios fueron realizados de forma unipodal en superficies estables. La tercera y cuarta semana los ejercicios se llevaron a cabo de forma unipodal y se agregaron actividades en superficies inestables para las tareas de equilibrio. Finalmente, en la quinta y sexta semana además de realizar los ejercicios unipodales, las tareas motoras sobre las superficies inestables fueron realizadas con los ojos cerrados.

\section{Entrenamiento convencional de basquetbol}

Los entrenamientos regulares de las deportistas se realizaban en el gimnasio del club deportivo. Tanto el grupo control como experimental asistían a 2 entrenamientos a la semana, en días no consecutivos. El entrenamiento de basquetbol, de acuerdo con el programa desarrollado por el equipo de preparación física del club, consistía en calentamiento, trabajo físico, trabajo táctico, enfriamiento y elongaciones. La duración de los entrenamientos fue de 90 minutos.

\section{Análisis estadístico}

Se utilizó el software estadístico SPSS versión 25.0 para el análisis de los resultados. Se calculó la media y desviación estándar para todas las variables. La distribución de los datos fue determinada con la prueba de Shapiro-Wilk. Se empleó la prueba de t-student para muestras relacionadas para comparar las evaluaciones pre y post intervención tanto en grupo control como en el grupo experimental. Para comparar el porcentaje de cambio entre los grupos se realizó la prueba t-student para muestras independientes. El tamaño del efecto se calculó con la $d$ de Cohen, considerando un efecto pequeño (e»0,2), moderado (e»0.5) o fuerte (e»0.8). Se consideró un nivel alfa de 0,05 para todos los análisis.

\section{Resultados}

De las 27 participantes de este estudio, 2 basquetbolistas no fueron consideradas en el análisis final. Una de las deportistas perteneciente al grupo experimental fue excluida por no cumplir con el porcentaje de asistencia a las sesiones de entrenamiento neuromuscular, mientras que otra participante del grupo control no fue considerada por una lesión sufrida durante el campeonato regular. Por lo tanto, la muestra analizada en este estudio corresponde a 25 jugadoras de basquetbol (12 grupo control y 13 grupo experimental). La media de las características generales de las participantes que conformaron el grupo control fue de 15,66 \pm 1,15 años, 60,25 \pm 8,99 $\mathrm{Kg}$ de peso, 1,64 \pm 0,08 m de estatura y 22,06 $\pm 1,93$ de IMC. Mientras que en el grupo de entrenamiento neuromuscular fue de 15,38 \pm 0,56 años, 59,38 \pm 7,20 Kg de peso, 1,70 \pm 0,05 m de estatura y 20,36 $\pm 1,46$ de IMC. No se presentaron diferencias significativas en estas variables entre los gru$\operatorname{pos}(p>0,05)$.

\section{Equilibrio postural dinámico}

En la tabla 1 y 2 se muestran los resultados del rendimiento en el SEBTm pre y post intervención tanto para el grupo control como experimental, respectivamente. En el grupo control se observó un aumento del rendimiento en la mayoría de las direcciones evaluadas tanto para la extremidad dominante como no dominante. Los aumentos estadísticamente significativos fueron 
encontrados en las direcciones: anterior de la extremidad dominante $(p=0,007 ; \mathrm{TE}=0,74)$, PL de la extremidad dominante $(p=0,002 ; \mathrm{TE}=0,31)$, anterior de la extremidad no dominante $(p=0,036 ; \mathrm{TE}=0,59) \mathrm{y}$ PL de la extremidad no dominante $(p=0,046$; TE $=$ $0,74)$. Los mayores cambios se observaron en la dirección anterior de la extremidad dominante con un aumento del 7,15\% y en la dirección PL en la extremidad no dominante con un cambio de un 7,77\% (tabla 1).

Tabla 1:

\begin{tabular}{|c|c|c|c|c|c|c|c|}
\hline \multirow[b]{2}{*}{ Direcciones SEBTm } & \multicolumn{2}{|c|}{ PRE } & \multicolumn{2}{|c|}{ POST } & \multirow{2}{*}{ Valor $\mathrm{p}$} & \multirow{2}{*}{$\begin{array}{c}\% \\
\text { Cambio }\end{array}$} & \multirow{2}{*}{ TE } \\
\hline & Media & $\mathrm{DE}$ & Media & $\mathrm{DE}$ & & & \\
\hline \multicolumn{8}{|l|}{ Extremidad dominante } \\
\hline Anterior & 60,25 & 5,54 & 64,56 & 6,09 & $0,007 *$ & 7,15 & 0,74 \\
\hline PM & 91,52 & 12,44 & 95,21 & 10,71 & 0,204 & 4,03 & 0,49 \\
\hline PL & 87,97 & 9,8 & 93,69 & 9,38 & $0,002 *$ & 6,50 & 0,31 \\
\hline Extremidad no dominante & & & & & & & 0,22 \\
\hline Anterior & 62,84 & 5,61 & 65,53 & 5,33 & $0,036^{*}$ & 4,28 & 0,59 \\
\hline PM & 96,44 & 9,72 & 94,33 & 8,84 & 0,327 & $-2,19$ & 0,81 \\
\hline PL & 86,86 & 9,82 & 93,61 & 6,41 & $0,046^{*}$ & 7,77 & 0,74 \\
\hline
\end{tabular}

Para el grupo de entrenamiento neuromuscular se observó un aumento tanto en la extremidad dominante como no dominante. Como se puede apreciar en la tabla 2, la extremidad dominante se reflejan cambios en todas sus direcciones: anterior $(p=0,001 ; \mathrm{TE}=2,20)$, $\mathrm{PM}(p=0,001 ; \mathrm{TE}=1,07)$ y $\mathrm{PL}(p=0,001 ; \mathrm{TE}=$ $0,99)$, mientras que en la extremidad no dominante los cambios se reflejaron en las direcciones anterior $(p=$ $0,001 ; \mathrm{TE}=1,50), \mathrm{PM}(p=0,007 ; \mathrm{TE}=0,57)$ y PL $(p$ $=0,006 ; \mathrm{TE}=0,64)$. El mayor cambio se evidencio en el alcance anterior de la extremidad dominante $(21,03 \%)$ (Tabla 2).

Tabla 2:

Resultados de balance postural dinámico pre y post intervención en grupo de entrenamiento neuromuscular.

\begin{tabular}{|c|c|c|c|c|c|c|c|}
\hline \multirow[b]{2}{*}{ Direcciones SEBTm } & \multicolumn{2}{|c|}{ PRE } & \multicolumn{2}{|c|}{ POST } & \multirow{2}{*}{ Valor $\mathrm{p}$} & \multirow{2}{*}{$\%$} & \multirow{2}{*}{ TE } \\
\hline & Media & $\mathrm{DE}$ & Media & $\mathrm{DE}$ & & & \\
\hline \multicolumn{8}{|l|}{ Extremidad dominante } \\
\hline Anterior & 57,73 & 6,31 & 69,87 & 4,59 & $0,001 *$ & 21,03 & 2.20 \\
\hline PM & 84,08 & 11,91 & 94,90 & 7,69 & $0,001 *$ & 12,87 & 1.07 \\
\hline PL & 81,34 & 13,81 & 93,17 & 9,57 & $0,001 *$ & 14,54 & 0.99 \\
\hline \multicolumn{8}{|c|}{ Extremidad no dominante } \\
\hline Anterior & 60,49 & 5,08 & 67,86 & 4,69 & $0,001 *$ & 12,18 & 1.50 \\
\hline PM & 85,88 & 13,85 & 93,53 & 12,58 & $0,007 *$ & 8,91 & 0.57 \\
\hline Posterolateral & 85,48 & 15,50 & 94,39 & 11,82 & $0,006 *$ & 10,42 & 0.64 \\
\hline
\end{tabular}

\section{Prueba de reposicionamiento activo}

En la tabla 3 y 4 se muestran los resultados del reposicionamiento activo de las articulaciones de extremidad inferior dominante como no dominante pre y post intervención para el grupo control y grupo de entrenamiento neuromuscular, respectivamente. En el grupo control no se observaron cambios significativos en las articulaciones evaluadas como se muestra en la tabla 3 .

En el grupo de entrenamiento neuromuscular, luego de la intervención, se observaron cambios en la
Tabla 3:

Resultados del reposicionamiento activo pre y post intervención en grupo control.

\begin{tabular}{|c|c|c|c|c|c|c|c|}
\hline \multirow[b]{2}{*}{ Articulaciones } & \multicolumn{2}{|c|}{ PRE } & \multicolumn{2}{|c|}{ POST } & \multirow{2}{*}{ Valor P } & \multirow{2}{*}{$\begin{array}{c}\% \\
\text { Cambio } \\
\end{array}$} & \multirow[b]{2}{*}{$\mathrm{TE}$} \\
\hline & Media & $\mathrm{DE}$ & Media & $\mathrm{DE}$ & & & \\
\hline \multicolumn{8}{|c|}{ Extremidad dominante } \\
\hline Cadera & 6,00 & 4,24 & 6,08 & 4,18 & 0,935 & 1,33 & 0.01 \\
\hline Rodilla & 5,41 & 2,57 & 4,5 & 2,54 & 0,204 & $-16,82$ & 0.35 \\
\hline Tobillo & 3,50 & 2,77 & 2,91 & 1,72 & 0,573 & $-16,86$ & 0.25 \\
\hline \multicolumn{8}{|c|}{ Extremidad no dominante } \\
\hline Cadera & 4,08 & 2,87 & 3,75 & 3,72 & 0,789 & $-8,09$ & 0.09 \\
\hline Rodilla & 5,00 & 4,80 & 3,08 & 3,57 & 0,072 & $-38,40$ & 0.45 \\
\hline Tobillo & 3,25 & 2,73 & 2,50 & 1,24 & 0,267 & $-23,08$ & 0.35 \\
\hline
\end{tabular}

mayoría de las articulaciones evaluadas. En la extremidad inferior dominante, el cambio significativo se encontró en las articulaciones de cadera $(p=0,001$; TE $=$ $2,72)$, rodilla $(p=0,001 ; \mathrm{TE}=2,70)$ y tobillo $(p=$ $0,001 ; \mathrm{TE}=2,07)$. Mientras que en la extremidad inferior no dominante los cambios fueron en las articulaciones de cadera $(p=0,028 ; \mathrm{TE}=0,97)$ y rodilla $(p=$ $0,023$; TE $=0,88)$. Los mayores cambios se evidenciaron en la articulación de cadera y rodilla de la extremidad dominante con un $-82,8 \%$ y $-77,4 \%$, respectivamente (Tabla 4).

Tabla 4:

Resultados del reposicionamiento activo pre y post intervención en grupo de entrenamiento neuromuscular.

\begin{tabular}{|c|c|c|c|c|c|c|c|}
\hline \multirow[b]{2}{*}{ Articulaciones } & \multicolumn{2}{|c|}{ PRE } & \multicolumn{2}{|c|}{ Post } & \multirow{2}{*}{ Valor P } & \multirow{2}{*}{$\%$ Cambio } & \multirow{2}{*}{$\mathrm{TE}$} \\
\hline & Media & $\mathrm{DE}$ & Media & DE & & & \\
\hline \multicolumn{8}{|c|}{ Extremidad dominante } \\
\hline Cadera & 7,15 & 2,96 & 1,23 & 0,83 & $0,001 *$ & $-82,80$ & 2.72 \\
\hline Rodilla & 8,15 & 3,18 & 1,84 & 0,89 & $0,001 *$ & $-77,42$ & 2.70 \\
\hline Tobillo & 7,38 & 3,73 & 1,76 & 0,83 & $0,001 *$ & $-76,15$ & 2.07 \\
\hline \multicolumn{8}{|c|}{ Extremidad no dominante } \\
\hline Cadera & 3,61 & 2,46 & 1,76 & 1,09 & $0,028^{*}$ & $-51,25$ & 0.97 \\
\hline Rodilla & 4,00 & 2,97 & 2,00 & 1,22 & $0,023 *$ & $-50,00$ & 0.88 \\
\hline Tobillo & 2,07 & 1,25 & 1,38 & 1,12 & 0,121 & $-33,33$ & 0.58 \\
\hline
\end{tabular}

\section{Comparación entre grupos}

Con respecto al equilibrio postural dinámico, la comparación del porcentaje de cambio pre y post intervención entre el grupo control y experimental determinó que el cambio fue significativamente mayor en el grupo de entrenamiento neuromuscular en las direcciones anterior $(p=0,001)$, posteromedial $(p=0,001) \mathrm{y}$ posterolateral $(p=0,004)$ del SEBTm para la extremidad dominante, mientras que para la extremidad no dominante el cambio del grupo de entrenamiento neuromuscular también fue mayor proporción al grupo control en las direcciones anterior $(p=0,006) \mathrm{y}$ posteromedial $(p=0,001)$ (Figura 1).

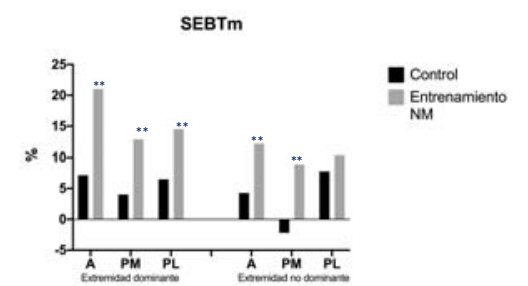

Figura 1. Comparación del porcentaje de cambio en el rendimiento del equilibrio dinámico (SEBTm) entre el grupo control y grupo de entrenamiento neuromuscular (NM). Se observa un mayor cambio en todas las direcciones del SEBTm evaluadas en el grupo de entrenamiento neuromuscular, tanto en extremidad dominante como no dominante. ${ }^{*} \mathrm{p}<0,05 ; * * \mathrm{p}<0,01$ 
Por su parte, en el reposicionamiento activo se observaron mayores cambios en el grupo de entrenamiento neuromuscular en comparación con el grupo control en todas las articulaciones de la extremidad dominante: cadera $(p=0,001)$, rodilla $(p=0,001)$ y tobillo $(p=$ $0,003)$. Para la extremidad no dominante se observó el mismo resultado al comparar el grupo de entrenamiento neuromuscular versus el grupo control: cadera $(p=$ $0,001)$, rodilla $(p=0,042)$ y tobillo $(p=0,033)$ (Figura 2).

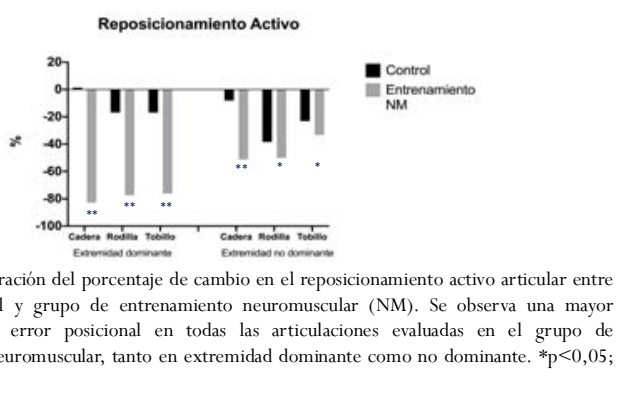

\section{Discusión}

El principal resultado de esta investigación señala que un entrenamiento neuromuscular de seis semanas de duración mejora el equilibrio postural dinámico y propiocepción de miembro inferior en basquetbolistas juveniles con IFT. Con respecto al equilibrio postural, hallazgos similares han sido reportados por otras investigaciones que muestran que el entrenamiento neuromuscular favorece su rendimiento tanto en pruebas estáticas como dinámicas en deportistas (GuzmánMuñoz et al., 2019; jung et al., 2014; Mendez-Rebolledo et al., 2021). Mientras que, en relación con la propiocepción, estudios previos muestran que un entrenamiento neuromuscular en deportistas favorece el rendimiento en pruebas como el reposicionamiento activo articular (Ghaderi, Letafatkar, Almonroeder, \& Keyhani, 2020; Mendez-Rebolledo et al., 2021).

En nuestro estudio las deportistas evaluadas presentaban una condición musculoesquelética asociada a un déficit neuromuscular de tobillo. Este déficit en personas con IFT ha sido atribuido al daño de los receptores sensoriales provocado por lesiones ligamentosas recurrentes (Freeman et al., 1965). Se ha establecido que el déficit de la información sensorial no es exclusivamente debido a la injuria del ligamento, sino que existen receptores capsulares, musculotendinosos y cutáneos que también se encuentran dañados (Riemann, Myers, Stone, \& Lephart, 2004). Estos receptores son mecanorreceptores y propioceptores que al encontrar- se dañados sensan ineficientemente la información relacionada con la tensión muscular, sensación del movimiento y sensación de la posición articular (Bruyneel, 2016). La información distorsionada proveniente de los propioceptores se procesa en el sistema nervioso central y provoca respuestas musculares inadecuadas (Jay Hertel, 2008). Por lo tanto, el déficit propioceptivo observado en personas con IFT afecta directamente el rendimiento de habilidades motoras como el equilibrio postural.

El mejor desempeño observado en la prueba de reposicionamiento activo articular en las basquetbolistas con IFT, posterior a 6 semanas de entrenamiento neuromuscular, se puede atribuir a principalmente a adaptaciones neurales centrales y periféricas que favorecen el control motor (Asadi, de Villarreal, \& Arazi, 2015). El sentido de la posición articular, como parte del componente propioceptivo, proporciona información sobre la posición de las articulaciones en el espacio (Bruyneel, 2016), por lo que el entrenamiento neuromuscular es fundamental para estimular los mecanorreceptores y propioceptores articulares que inducen estas adaptaciones (Asadi et al., 2015). Las adaptaciones periféricas inducidas por el entrenamiento neuromuscular se deben probablemente a la estimulación repetitiva de estos receptores articulares en movimientos sobre superficies inestables y sin input visual (SilvaMoya et al.). Por otro lado, las adaptaciones centrales podrían ocurrir a través del aprendizaje motor y la automaticidad de los movimientos que inducirían la programación motora a nivel cerebral (Voight \& Wieder, 1991).

Asimismo, en esta investigación se pudo observar que un entrenamiento neuromuscular promueve un cambio beneficioso del equilibrio postural. Dentro de este tipo de entrenamiento el trabajo de la estabilidad dinámica se transforma en un contenido esencial utilizado en la planificación de los programas de intervenciones neuromusculares (Sanudo et al., 2019). La evidencia respalda la necesidad de entrenar la estabilidad dinámica debido a las mejoras en el sistema sensoriomotor que permiten mejorar el control neuromuscular (Sanudo et al., 2019). El propósito de este mecanismo es estimular la actividad propioceptiva y dar retroalimentación a los centros integradores para generar respuestas musculares apropiadas (MendezRebolledo et al., 2015). Esto conduce a una mejor función propioceptiva y, por tanto, a un mejor equilibrio postural (Sanudo et al., 2019). La literatura muestra que el entrenamiento neuromuscular para personas con 
IFT debe entrenarse a través de ejercicios enfocados en las extremidades inferiores y el CORE (Sanudo et al., 2019), utilizando superficies estables e inestables y con actividades con ojos abiertos y cerrados, lo cual contribuye a desafiar este mecanismo de retroalimentación (Guzmán-Muñoz et al., 2019). Esto se debe combinar con actividades de aterrizaje, desaceleración y cambios de dirección para estimular acciones anticipadas que ocurren antes de que el sistema sensoriomotor detecte cambios en el entorno (Sanudo et al., 2019). Es por esto que la planificación del entrenamiento neuromuscular desarrollado en este estudio incorporó todos los principios anteriormente descritos y se reflejó en una mejor propiocepción y equilibrio postural luego de 6 semanas de intervención. Además, las diferencias observadas entre el grupo control y experimental en el porcentaje de cambio revela el impacto beneficioso de incorporar actividades neuromusculares al entrenamiento de los deportistas.

Dentro de las limitaciones de nuestro estudio se encuentra la falta de un período de seguimiento. Este factor es fundamental para conocer los efectos reales de las terapias a mediano y largo plazo. Se sugiere que se incluya el seguimiento de los grupos para futuras investigaciones.

\section{Conclusión}

En conclusión, esta investigación señala que un entrenamiento neuromuscular de 6 semanas mejoró la propiocepción, específicamente la sensación de posición articular, y favoreció el equilibrio postural en basquetbolistas con IFT. Este tipo de entrenamiento busca brindar información adecuada a los mecanoreceptores y propioceptores para que la integración de las respuestas musculares sea más eficiente. Por ello, sugerimos incluir, en la actividad física de los deportistas, actividades motoras y ejercicios que incluyan tareas de equilibrio, propiocepción y fuerza muscular en superficies estables e inestables y con ausencia de input visual. Esto podría promover una mayor estabilidad articular, mejor rendimiento deportivo y disminuir el riesgo de lesión de tobillo.

\section{Referencias}

Asadi, A., Saez de Villarreal, E., \& Arazi, H. (2015). The Effects of Plyometric Type Neuromuscular Training on Postural Control Performance of Male Team Basketball Players. Journal of strength and conditioning research, 29(7), 1870-1875. https: / / doi.org/10.1519/JSC.0000000000000832

Bruyneel, A. V. (2016). Evaluación de la propiocepción: pruebas de estatestesia y cinestesia. EMCKinesiterapia-Medicina Física, 37(4), 1-11. https: / / doi.org/10.1016/S1293-2965(16)78903-1

Danes Daetz, C., Rojas Toro, F., \& Tapia Mendoza, V. (2020). Lesiones deportivas en deportistas universitarios chilenos (Sports injuries in Chilean university athletes). Retos, 38, 490-496. https://doi.org/ $10.47197 /$ retos.v38i38.74745

De Oliveira Menacho, M., Pereira, H. M., Oliveira, B. I., Chagas, L. M., Toyohara, M. T., \& Cardoso, J. R. (2010). The peroneus reaction time during sudden inversion test: systematic review. Journal of electromyography and kinesiology : official journal of the International Society of Electrophysiological Kinesiology, 20(4), 559-565. https://doi.org/ 10.1016/j.jelekin.2009.11.007

Fatima, S., Bhati, P., Singla, D., Choudhary, S., \& Hussain, M. E. (2020). Electromyographic Activity of Hip Musculature During Functional Exercises in Participants With and Without Chronic Ankle Instability. Journal of chiropractic medicine, 19(1), 8290. https: / / doi.org/10.1016/j.jcm.2019.07.002

Fong, D. T., Hong, Y., Chan, L. K., Yung, P. S., \& Chan, K. M. (2007). A systematic review on ankle injury and ankle sprain in sports. Sports medicine (Auckland, N.Z.), 37(1), 73-94. https://doi.org/10.2165/ 00007256-200737010-00006

Freeman, M. A., Dean, M. R., \& Hanham, I. W. (1965). The etiology and prevention of functional instability of the foot. The Journal of bone and joint surgery. British volume, 47(4), 678-685.

Ghaderi, M., Letafatkar, A., Almonroeder, T. G., \& Keyhani, S. (2020). Neuromuscular training improves knee proprioception in athletes with a history of anterior cruciate ligament reconstruction: A randomized controlled trial. Clinical biomechanics (Bristol,Avon), 80, 105157.

Gribble, P. A., Hertel, J., \& Plisky, P. (2012). Using the Star Excursion Balance Test to assess dynamic postural-control deficits and outcomes in lower extremity injury: a literature and systematic review. Journal of athletic training, 47(3), 339-357. https: / /doi.org/10.4085/1062-6050-47.3.08

Gribble, P. A., Kelly, S. E., Refshauge, K. M., \& Hiller, C. E. (2013). Interrater reliability of the star excursion balance test. Journal of athletic training, 48(5), 621-626. https:/ / doi.org/10.4085/ 
1062-6050-48.3.03

Guzmán-Muñoz, E. , Daigre-Prieto, M., Soto-Santander, K., Concha-Cisternas, Y., Méndez-Rebolledo, G., Sazo-Rodríguez, S., \& Valdés-Badilla, P. (2019). The effects of neuromuscular training on the postural control of university volleyball players with functional ankle instability: A pilot study. Archivos de medicina del deporte, 35(5), 283-287.

Guzmán-Muñoz, E., Sazo-Rodriguez, S., Concha-Cisternas, Y., Valdés-Badilla, P., Lira-Cea, C., SilvaMoya, G., Henríquez, R., Farias, T.Y., Cigarroa, I., Castillo-Retamal, M., \& Méndez-Rebolledo, G. (2020). Four Weeks of Neuromuscular Training Improve Static and Dynamic Postural Control in Overweight and Obese Children: A Randomized ControlledTrial. Journal of motor behavior, 52(6), 761769. https://doi.org/10.1080/ 00222895.2019 .1694486

Hertel J. (2008). Sensorimotor deficits with ankle sprains and chronic ankle instability. Clinics in sports medicine, 27(3), 353-vii. https: / / doi.org/10.1016/ j.csm.2008.03.006

Hertel, J., Braham, R. A., Hale, S. A., \& OlmstedKramer, L. C. (2006). Simplifying the star excursion balance test: analyses of subjects with and without chronic ankle instability. The Journal of or thopaedic and sports physical therapy, 36(3), 131-137. https: / / doi.org/10.2519/jospt.2006.36.3.131

Horak F. B. (2006). Postural orientation and equilibrium: what do we need to know about neural control of balance to prevent falls?. Age and ageing, 35 Suppl 2, ii7-ii11. https: / / doi.org/10.1093/ageing/afl077

Iida, N., Kaneko, F., Aoki, N., \& Shibata, E. (2014). The effect of fatigued internal rotator and external rotator muscles of the shoulder on the shoulder position sense. Journal of electromyography and kinesiology : official journal of the International Society of Electrophysiological Kinesiology, 24(1), 72-77. https: / /doi.org/10.1016/j.jelekin.2013.10.008

Jung, D. J., Kang, J. I., Park, S. K., Lee, M. K., \& Jeong, Y. S. (2014). Effect of Neuromuscular Training on Postural Control and Jump Performance in Functional Ankle Instability Soccer Player. Korean Journal of Sport Biomechanics, 24(3), 295-300. https://doi.org/ 10.5103/kjsb.2014.24.3.295

Karagiannopoulos, C., Sitler, M., Michlovitz, S., Tucker, C., \& Tierney, R. (2016). Responsiveness of the active wrist joint position sense test after distal radius fracture intervention. Journal of hand therapy : official journal of the American Society of Hand Therapists, 29(4), 474-482.https://doi.org/10.1016/ j.jht.2016.06.009

Lin, C. W., Delahunt, E., \& King, E. (2012). Neuromuscular training for chronic ankle instability. Physical therapy, 92(8), 987-991. https:/ /doi.org/10.2522/ptj.20110345

Lubiatowski, P., Ogrodowicz, P., Wojtaszek, M., \& Romanowski, L. (2019). Bilateral shoulder proprioception deficit in unilateral anterior shoulder instability. Journal of shoulder and elbow surgery, 28(3), 561-569. https://doi.org/10.1016/ j.jse.2018.08.034

Matsouka, O., Nani, S., Papadimitriou, K., Astrapellos, K., Beneka, A., \& Malliou, P. (2020). Time course changes in hand grip strength performance and hand position sense in climbing. Journal of Human Sport and Exercise, 15(1), 23-33. https: / / doi.org/10.14198/ jhse.2020.151.03

Maurer, C., Mergner, T., \& Peterka, R. J. (2006). Multisensory control of human upright stance. Experimental Brain Research, 171(2), 231-250. https:// doi.org/10.1007/s00221-005-0256-y

McGuine, T. A., Greene, J. J., Best, T., \& Leverson, G. (2000). Balance as a predictor of ankle injuries in high school basketball players. Clinical Journal of Sport Medicine, 10(4), 239-244. https: / / doi.org/10.1097/ 00042752-200010000-00003

Mendez-Rebolledo, G., Figueroa-Ureta, R., MoyaMura, F., Guzmán-Muñoz, E., Ramirez-Campillo, R., \& Lloyd, R. S. (2021). The Protective Effect of Neuromuscular Training on the Medial Tibial Stress Syndrome in Youth Female Track-and-Field Athletes: A Clinical Trial and Cohort Study. Journal of sport rehabilitation, 1-9. Advance online publication. https://doi.org/10.1123/jsr.2020-0376

Mendez-Rebolledo, G., Guzman-Munoz, E., GaticaRojas, V., \& Zbinden-Foncea, H. (2015). Longer reaction time of the fibularis longus muscle and reduced postural control in basketball players with functional ankle instability: A pilot study. Physical Therapy in Sport, 16(3), 242-247. https: / / doi.org/ 10.1016/j.ptsp.2014.10.008

Peterka, R. J., \& Loughlin, P. J. (2004). Dynamic regulation of sensorimotor integration in human postural control. Journal of Neurophysiology, 91(1), 410423. https:/ /doi.org/10.1152/jn.00516.2003

Rein, S., Hagert, E., \& Sterling-Hauf, T. (2021). Alterated ligamento-muscular reflex pattern after stimulation of the anterior talofibular ligament in functional ankle instability. Knee surgery, sports 
traumatology, arthroscopy : official journal of the ESSKA, 29(5), 1544-1553. https://doi.org/ 10.1007/s00167-020-06232-w

Riemann, B. L., Myers, J. B., Stone, D. A., \& Lephart, S. M. (2004). Effect of lateral ankle ligament anesthesia on single-leg stance stability. Medicine and Science in Sports and Exercise, 36(3), 388-396. https: / /doi.org/10.1249/01.mss.0000117131.93989.9b

Rodriguez-Fernandez, A. L., Rebollo-Roldan, J., Jimenez-Rejano, J. J., \& Gueita-Rodriguez, J. (2015). Psychometric properties of the Spanish version of the Cumberland Ankle Instability Tool. Disability and Rehabilitation, 37(20), 1888-1894. https: / / doi.org/ 10.3109/09638288.2014.984879

Sanchez-Monzó, C. S., Lanzuela, M. F., \& Alfaro, J. J. B. (2015). Inestabilidad crónica de tobillo: Actualización. Revista de la Sociedad Andaluza de Traumatología y Ortopedia, 32(2), 19-29.

Sanudo, B., Sanchez-Hernandez, J., Bernardo, M., Abdi, E., Taiar, R., \& Nunez, J. (2019). Integrative Neuromuscular Training in Young Athletes, Injury Prevention, and Performance Optimization: A Systematic Review. Applied Sciences-Basel, 9(18). https: / / doi.org/10.3390/app9183839

Silva-Moya, G., Méndez-Rebolledo, G., Valdes-Badilla, P., Gómez-Álvarez, N., \& Guzmán-Muñoz, E. (2021). Effects of neuromuscular training on psychomotor development and active joint position sense in school children. Journal of motor behavior, 1-10. Advance online publication. https://doi.org/10.1080/

\subsection{1 .1887072}

Steib, S., Rahlf, A. L., Pfeifer, K., \& Zech, A. (2017). Dose-Response Relationship of Neuromuscular Training for Injury Prevention in Youth Athletes: A Meta-Analysis. Frontiers in physiology, 8, 920. https:/ /doi.org/10.3389/fphys.2017.00920

Toro Román, V., Guerrero Ramos, D., Muñoz Marín, D., Siquier Coll, J., Bartolomé Sánchez, I., \& Robles Gil, M. (2020). Análisis de la incidencia de lesiones y hábitos usados durante el calentamiento en el baloncesto femenino (Analysis of the incidence of injuries and routines used during warm-up in female basketball players). Retos, 38, 159-165. https: // doi.org/10.47197/retos.v38i38.74310

Voight, M. L., \& Wieder, D. L. (1991). Comparative reflex response times of vastus medialis obliquus and vastus lateralis in normal subjects and subjects with extensor mechanism dysfunction. An electromyographic study. The American journal of sports medicine, 19(2), 131-137. https://doi.org/10.1177/ 036354659101900207

Wenning, M., Gehring, D., Mauch, M., Schmal, H., Ritzmann, R., \& Paul, J. (2020). Functional deficits in chronic mechanical ankle instability. Journal of orthopaedic surgery and research, 15(1), 304. https: / / doi.org/10.1186/s13018-020-01847-8

Yeung, M. S., Chan, K. M., So, C. H., \& Yuan, W. Y. (1994). An epidemiological survey on ankle sprain. British journal of sports medicine, 28(2), 112116. https:/ /doi.org/10.1136/bjsm.28.2.112

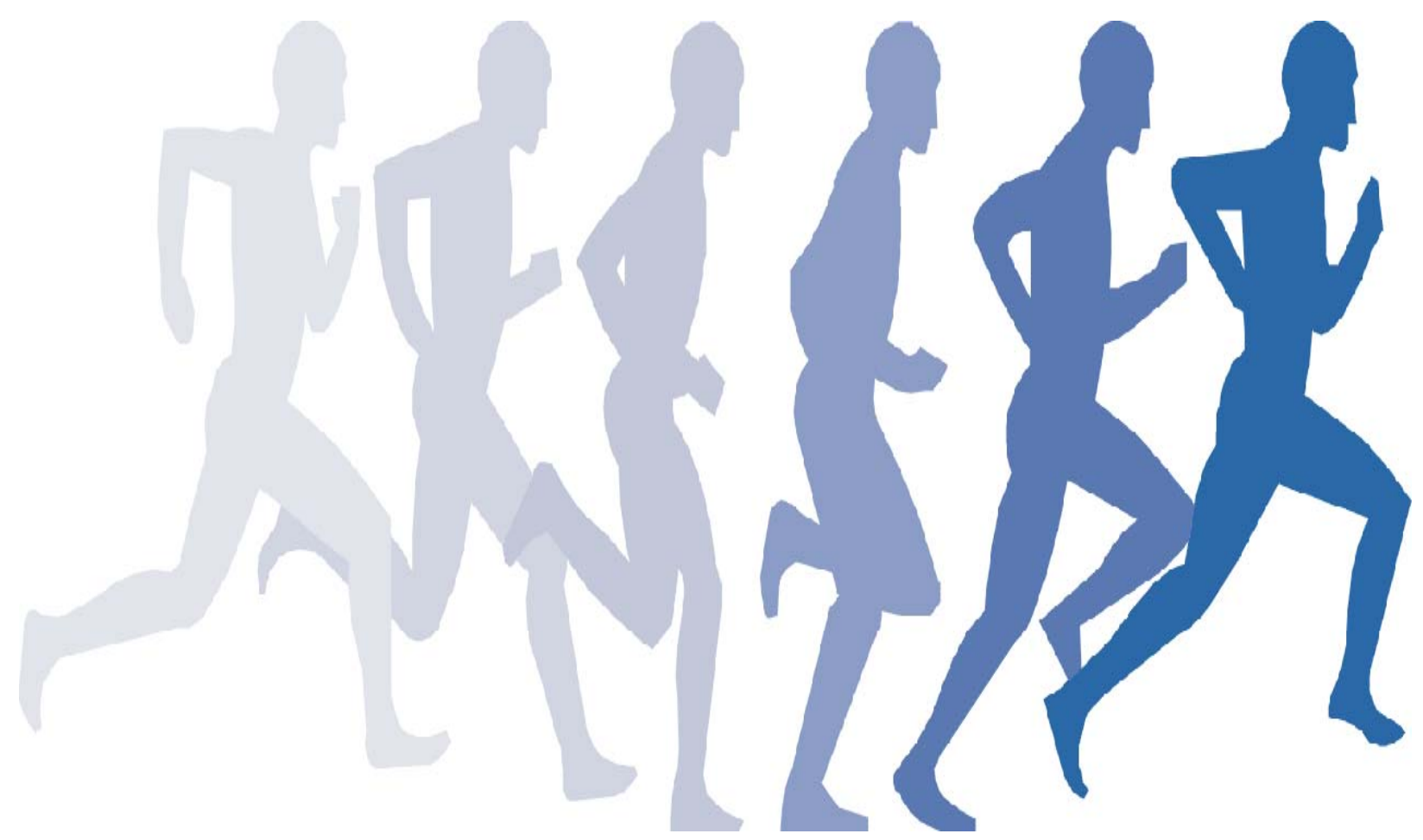

